\title{
Artrogripose Múltipla Congênita: Relato de dois casos
}

Dagna Karen Oliveira $^{1}$ (D), Bruna Fernandes ${ }^{1}$ (D), Amanda Alencar dos Anjos ${ }^{1}$ (D), Sadana Hillary Dal'Negro $^{1}$ (D), Dyayne Carla Banovski ${ }^{1}$ (D), Bárbara Louise Ferreira ${ }^{1}$ (D), Victoria Gimenes Freitas ${ }^{1}$ (D), Renata Bragato Futagami ${ }^{1}$ (D)

\begin{abstract}
RESUMO
A Artrogripose Múltipla Congênita (AMC) é uma síndrome rara, que representa um grupo de condições congênitas caracterizado por contraturas articulares em duas ou mais articulações, não progressivas, geralmente simétricas, de etiologia desconhecida e que pode estar associada a outras malformações, como viscerais e neurológicas. Apresentamos dois casos isolados com achados característicos de AMC, atendidos em ambulatório de pediatria de alto risco de uma cidade do Oeste do Paraná. Caso 1: lactente, sexo feminino, 14 dias de vida, com redução dos movimentos fetais referidos pela mãe, apresentando malformações envolvendo somente os membros, visualizadas logo após o nascimento. Caso 2: lactente, sexo masculino, quatro meses de vida, com diagnóstico de hipocinesia fetal ao ultrassom obstétrico de 23 semanas, ao nascimento apresenta contraturas dos membros e disfunção do sistema nervoso central. O diagnóstico da artrogripose é complexo e essencialmente clínico, sendo necessária uma anamnese meticulosa, contemplando uma avaliação da gestação, via de parto, história familiar e um exame físico minucioso, classificando quais os tipos de contraturas, membros envolvidos, presença de musculatura alterada e de alterações neurológicas, a fim de diferenciar a AMC das outras síndromes que também cursam com contraturas articulares. O seguimento e tratamento dos lactentes com artrogripose devem ocorrer em acompanhamento multidisciplinar devido às variadas manifestações que a doença pode apresentar e à característica de recidiva ao longo dos anos.
\end{abstract}

PALAVRAS-CHAVE: Artrogripose, Hipocinesia Experimental, Contraturas, Integralidade em Saúde.

\section{INTRODUÇÃO}

Síndrome rara e de etiologia desconhecida, a Artrogripose Múltipla Congênita (AMC) foi descrita por Otto como miodistrofia congênita em 1841, e denominada como AMC por Stern, em $1923^{1-3}$. Com uma prevalência estimada variando entre um a cada 3.300 e 12.000 nascidos vivos em ambos os sexos, a AMC é um termo usado para descrever um grupo de condições congênitas, caracterizada por várias contraturas articulares em duas ou mais articulações, não progressivas e geralmente simétricas ${ }^{4-5}$. Além do envolvimento dos membros, pode manifestar também assimetria facial, micrognatia, nariz em sela, pele delgada, atrofia muscular, escoliose, degenerações nos sistemas nervoso e urinário, hipoplasia pulmonar, intestino imaturo e/ou curto e osteoporose de ossos longos ${ }^{5-7}$.

A etiopatogenia da AMC é multifatorial e, apesar de ainda ter a causa exata desconhecida por alguns profissionais, resulta de fatores genéticos, parentais, ambientais e/ou anormalidades durante o desenvolvimento fetal que atingem a mãe e o concepto, como: medicamentos, infecções, oligoidrâmnio, polidrâmnio, alteração uterina, doenças crônicas e traumas ${ }^{5,7}$.

Clinicamente a AMC é classificada por Hall ${ }^{7}$ em três categorias, em ordem de gravidade: envolvimento somente dos membros, envolvimento dos membros mais outra anormalidade e envolvimento dos membros com disfunção do sistema nervoso central (SNC). A cognição, que inclui aspectos de percepção, atenção, memória, bem como desenvolvimento da linguagem, pode ser afetada se o SNC estiver envolvido, devido à associação entre a AMC $e$ genes relacionados ao desenvolvimento e à diferenciação de células neurais ${ }^{8}$. A disfunção pode se manifestar através de atraso no desenvolvimento neuropsicomotor, microgiria, microcefalia, convulsões, hidrocefalia, lisencefalia e ausência do corpo caloso ${ }^{8}$. 
O objetivo deste estudo é relatar dois casos de artrogripose, destacar a AMC entre os diagnósticos diferenciais de outras síndromes que também evoluem com contraturas articulares, assim como enfatizar a importância do trabalho multidisciplinar no diagnóstico da doença e no prognóstico das crianças acometidas, visto que o tratamento adequado pode propiciar melhor qualidade de vida às crianças e a oportunidade de uma vida com menos limitações.

\section{RELATO DO CASO}

\section{CASO 1}

Lactente do sexo feminino, com 14 dias de vida, nascida em 2019, caucasiana, encaminhada ao ambulatório de alto risco por malformação das mãos e dos pés, visualizada logo após o parto, para acompanhamento multidisciplinar.

Mãe de 29 anos, terceira gestação, sem intercorrências, infecções gestacionais ou traumas, sendo antecedida por um parto cirúrgico e um aborto espontâneo de seis semanas gestacionais seguido de curetagem. Mãe, com histórico pessoal de síndrome de Legg-Calvé-Perthes e asma, em uso de corticoide inalatório, nega malformações uterinas e ausência de consanguinidade entre os pais.

História familiar de tia paterna com hidrocefalia e posterior óbito e natimortalidade em tio paterno, sem investigação de causa, em gestação gemelar tardia. Irmã mais velha não apresenta anormalidades congênitas ou de desenvolvimento.

Relato materno de pouca movimentação intrauterina, principalmente no terceiro trimestre. A ultrassonografia (USG) morfológica apresentou polidrâmnio. Recém-nascida (RN) de 39 semanas gestacionais, pesando $2,950 \mathrm{~kg}, 42 \mathrm{~cm}$ de comprimento, $36 \mathrm{~cm}$ de perímetro cefálico, Apgar 9 e 10, parto via cesariana. Triagem neonatal biológica ampliada, triagem auditiva neonatal e teste do reflexo vermelho sem alterações, cariótipo 46, XX.

Ao exame físico, massa muscular adequada para idade, sem necessidade de maiores investigações, quadris com limitação da abdução, pés com tálus vertical bilateral, rígidos e com limitação da flexão plantar. Mãos com deformidades, em flexão dorsal dos punhos, articulações inter- falangeanas estendidas, com desvio ulnar e micrognatia. Desenvolvimento neurológico adequado para a idade.

Ultrassonografia do quadril apresenta redução do ângulo alfa e radiografia de quadril mostra subluxação à direita. Radiografias das mãos direita e esquerda com deformidade de ossos da mão. Radiografias dos pés direito e esquerdo apresentam alterações da disposição de falanges do $2^{\circ}$ ao $5^{\circ}$ dedo com sobreposição dos dedos, sinais de pés talus verticalis e acentuação do arco plantar (Figura 1). Ecografia de abdome total e ecocardiograma sem alterações.
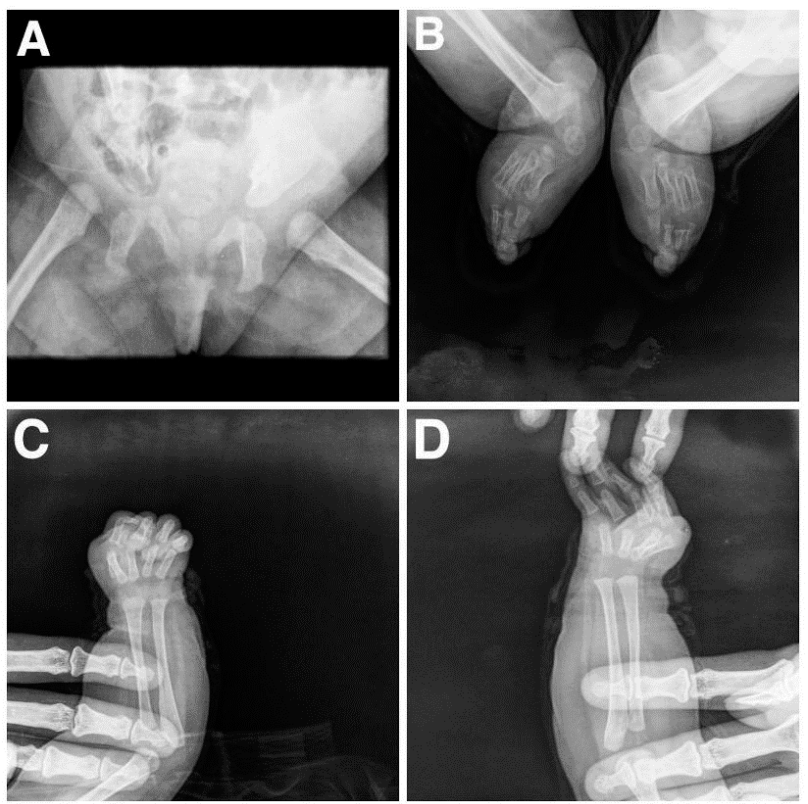

Figura 1. Radiografia do primeiro caso. A: Luxação superior do quadril direito, com sinais sugestivos de displasia do desenvolvimento do quadril. $\mathrm{B}$ : Membros inferiores apresentando sinais de pés talus verticalis e posição de flexão dos pododáctilos com acentuação do arco plantar. C: Membro superior direito em repouso. D: Membro superior direito com extensão passiva dos quirodáctilos. Ambas posições (C e D) apresentam contraturas fixas no punho e quirodáctilos.

RN encaminhada ao hospital de referência em pediatria da América Latina, onde foi confirmado o diagnóstico de artrogripose distal. A lactente fez uso de imobilização com gesso em membros inferiores por dois meses, em uso de órtese nas mãos, solicitada cirurgia de correção para os pés, procedimento em fila de espera. Após fisioterapia, apresenta melhora funcional. Aguarda avaliação com fonoaudióloga e buco-maxilo-facial. 


\section{CASO 2}

Lactente, sexo masculino, quatro meses e três dias de vida, encaminhado ao ambulatório de alto risco para atendimento multidisciplinar por diagnóstico de AMC.

História gestacional de USG morfológica de primeiro trimestre com translucência nucal alterada $(0,57 \mathrm{~mm})$. A segunda USG morfológica evidenciou hidropsia fetal, linfangioma do tipo higroma cístico e polidrâmnio. A gestante foi encaminhada para Curitiba, onde foram identificadas em USG acinesia fetal, micrognatia moderada e presença de contraturas: mãos fechadas, dedos da mão esquerda sobrepostos, coxas unidas e paralelas, com flexão sobre o tronco e hiperextensão dos joelhos, quadro compatível com AMC.

Mãe de 31 anos, terceira gestação e histórico de dois partos cesarianos, nega complicações gestacionais, traumas ou doenças infecciosas. Não faz uso álcool, tabaco ou outras drogas e nega doenças prévias, patologias gestacionais, alterações uterinas ou natimortalidade. Sem histórico familiar de malformação ou outras doenças. Relata-se parentesco de quarto grau de consanguinidade entre os progenitores.

Lactente, nascido em 2018 de parto cesariana, com 37 semanas gestacionais, $3,240 \mathrm{~kg}$, perímetro cefálico de $36 \mathrm{~cm}$, Apgar 5 e 7 (por hipoatividade e apneia), neonato sem reflexo de sucção e deglutição ao nascimento. Evidenciado presença de pequeno higroma cístico em região cervical, sem necessidade de investigação e intervenções. Triagem auditiva neonatal alterada, sendo solicitado exame Potencial Evocado Auditivo do Tronco Encefálico com resultado normal bilateralmente. Triagem neonatal biológica ampliada sem alterações e cariótipo de 46, XY. Exame de fundo de olho apresentou hemorragias pré-retinianas bilaterais e difusas em polo posterior, já na fundoscopia, presença de micro-hemorragia única em região macular, em reabsorção.

Encaminhado à Unidade de Terapia Intensiva Neonatal (UTIN), onde permaneceu por 45 dias, e necessitou de ventilação mecânica, sonda nasogástrica e posterior gastrostomia. Evidenciado forame oval patente, luxação de quadris e joelhos. Realizado eletroencefalograma que evidenciou atividade epileptiforme frequente nas regiões fronto-centro-temporais, sem evidências clínicas, tratadas com clonazepam e fenobarbital. Em tomografia computadorizada de crânio, presença de sinais de perda volumétrica cerebral, focos de hipoatenuação na substância branca periventricular e dos semiovais de aspectos inespecíficos.

Aos quatro meses de vida passa a sustentar os olhos abertos, entretanto, não realiza abertura e fechamento das mãos e possui restrição de movimentos em membros superiores. Não apresenta movimentação na articulação do joelho, move-se com ajuda da musculatura abdominal (Figura 2).
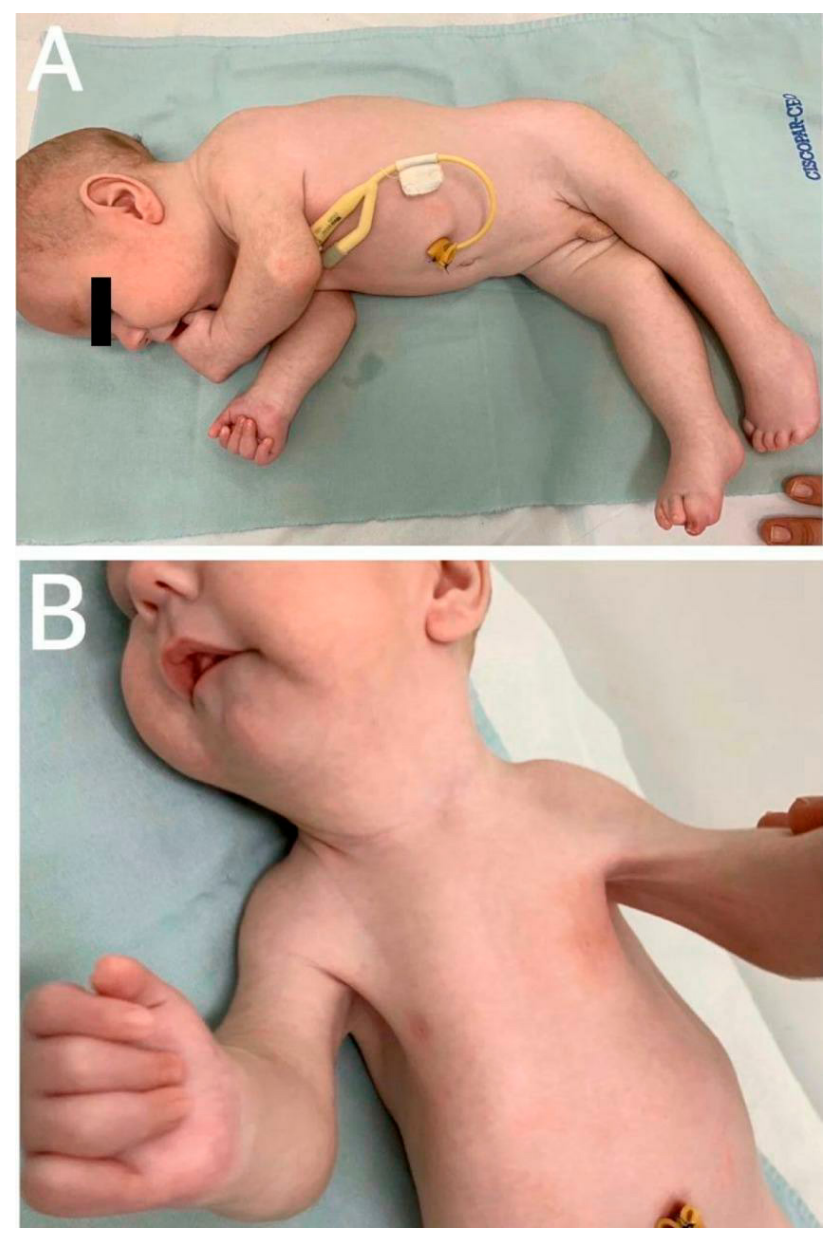

Figura 2. Paciente do segundo caso. A - Posição em repouso: verificam-se contraturas em membros superiores e alterações na disposição dos quirodáctilos. Nos membros inferiores, visualiza-se contratura fixa, extensão do quadril, joelhos hiperestendidos e sobreposição de pododáctilos. B - Membros superiores em extensão passiva: observam-se contraturas fixas e limitação de movimento. Nota-se, na figura, micrognatia em face e bolsa de gastrostomia.

A mãe referiu melhora da motricidade e da sustentação do pescoço após acompanhamento 
com fisioterapia e fonoterapia, porém, relata que aos 16 meses de vida o lactente realiza deglutição de pequenas doses de água ofertadas com seringa e não mantém a boca fechada. Lactente encaminhado ao Hospital Referência de Pediatria da América Latina, onde faz acompanhamento com gastroenterologista, neurologista, ortopedista e geneticista. Permanece em uso de clonazepam, fenobarbital e oxcarbazepina para tratamento das crises convulsivas tônico-clônica.

Ambas as crianças seguem em acompanhamento multidisciplinar pela Atenção Primária em conjunto com o ambulatório de alto risco Modelo de Atenção às Condições Crônicas (MACC).

De acordo com a carta circular n. ${ }^{0}$ 166/2018-CONEP/SECNS/MS, esse relato de dois casos foi aprovado pelo CEP/SCS/UFPR no parecer consubstanciado número 4.219.939.

\section{DISCUSSÃO}

A acinesia ou hipomobilidade fetal, principal achado etiológico da artrogripose, pode estar relacionada a fatores miogênicos, doenças dos tecidos adjacentes ao ambiente em que o feto se desenvolve, doenças maternas, fatores mecânicos, vasculares ou nutricionais e fatores neurogêni$\cos ^{5,7,9-10}$. A contratura articular é um sinal clínico presente em mais de 400 doenças, independentemente da patologia subjacente ao aparecimento dessa hipomobilidade ${ }^{5}$.

Há uma relação clínica de precocidade no início da hipomobilidade fetal e a gravidade das sequelas ${ }^{7}$. Associação afirmada nos relatos de casos descritos acima: no caso 1, a mãe sensibilizou a diminuição dos movimentos intra-útero no terceiro trimestre gestacional e obteve como diagnóstico artrogripose distal I, primeiro tipo da classificação de Hall; já no segundo caso, a mãe identificou a hipocinesia em período anterior às 23 semanas gestacionais e o neonato apresentou sequelas com maior gravidade, sendo classificado no terceiro tipo de Hall, devido ao envolvimento no $\mathrm{SNC}^{7,9,11}$.

Quando a artrogripose compromete exclusivamente os membros, é possível identificar como principais manifestações a flexão palmar com desvio ulnar da mão, pé talo vertical, luxação dos quadris e contratura dos dedos ${ }^{1,7,9}$. Nes- sas situações, a gravidade de cada situação de AMC depende, entre outras condições clínicas, de quantas articulações do corpo são afetadas e seu nível de rigidez ${ }^{5}$. Outras anormalidades não articulares também são associadas frequentemente com a artrogripose, como cardiopatias congênitas (forame oval patente) e distúrbios neurológicos (crises convulsivas), achados destacados no paciente do segundo caso, além das contraturas características de artrogripose $e^{5,10,12}$.

A AMC também pode provocar disfagia e dificuldade de deglutição nos pacientes que possuem sequelas como micrognatia ${ }^{13}$. A contração da articulação temporomandibular também é um fator comumente agravante ${ }^{14}$. Quando associadas, essas disfunções orofaríngeas podem impedir uma alimentação segura pela via oral pelo risco de aspiração, indicando a abertura de uma via alternativa ${ }^{13,14}$. No caso 2 , a gastrostomia foi prescrita a fim de fornecer uma via de nutrição enteral de longo prazo adequada ao paciente ${ }^{14}$. Ademais, no seguimento desses pacientes, é necessária a investigação de outros problemas, como respiratórios, estéticos e na fala, comuns nos portadores de micrognatismo, de forma a ampliar não só a qualidade de vida, como, também, suas sobrevidas ${ }^{10,15}$.

O diagnóstico da artrogripose é complexo e essencialmente clínico, devido à grande variedade de síndromes que podem cursar com contraturas articulares ${ }^{12}$. Cerca de $75 \%$ das vezes o diagnóstico é feito tardiamente (após o nascimento), como constatado no caso 1 , possivelmente devido à falta de pesquisa sobre movimentação fetal durante o pré-natal, fato que retarda o início precoce do tratamento e prejudica o prognóstico ${ }^{7,12}$. Para uma avaliação completa, é necessário investigar a gestação, via de parto, história familiar e um exame físico minucioso, principalmente visando classificar quais os tipos de contraturas, membros envolvidos, postura de flexão ou extensão, presença de musculatura alterada e de alterações neurológicas ${ }^{4,9,12}$.

A gama de diagnósticos diferenciais para a artrogripose é extensa, e exames como radiografias, testes genéticos, biópsias musculares e exames de sangue podem ajudar a excluir outras causas quando o diagnóstico apenas clínico for de difícil conclusão $0^{15}$. Entre essas patologias diferenciais estão a síndrome de Larsen, síndrome de Escobar, os- 
teogênese imperfeita, síndrome do alcoolismo fetal, mielomeningocele, síndrome de Turner, trissomia do 21 , entre outras ${ }^{9}$. A exclusão de diagnósticos é realizada por exames e detalhes clínicos característicos de cada doença. Em casos de dúvidas, a AMC deve ser avaliada como diagnóstico de exclusão, descartando outras causas. Para excluir a síndrome de Escobar, por exemplo, além das contraturas, devemos evidenciar a presença de sindactilias, fenda labial ou palatina e, mais classicamente, presença de pterígio cervical ${ }^{9,15}$.

Descrito em ambos os casos, o diagnóstico e a conduta precoce, via equipe multidisciplinar, é de importância singular, principalmente durante o primeiro ano de vida, período em que a criança estará se adaptando para começar a caminhar, minimizando as sequelas e o comprometimento da autonomia do lactente ${ }^{9,12}$.

O seguimento e tratamento da artrogripose devem ocorrer de forma criteriosa, devido às variadas manifestações que a doença pode apresentar, às múltiplas articulações que podem ser acometidas e à característica de recidiva ao longo dos anos, pois as contraturas e rigidez de tecidos periarticulares impedem um definitivo remodelamento osteoarticular, tornando necessário um acompanhamento prolongado com assistência de equipe multiprofissional ${ }^{1,7,9}$.

Em casos de AMC mais severos ou negligenciados, além de sessões de fisioterapia, do uso de órteses, imobilização com gesso e manuseio das deformidades, faz-se preciso a cirurgia de correção das deformações osteoarticulares em mãos, punhos, coluna, quadris, joelhos ou pés, buscando a independência de socialização e deambulação ao permitir a mobilidade de membros ${ }^{9,12}$.

A melhor conduta destinada ao portador de AMC inclui, em todas as fases da vida, a educação dos pais referente a natureza da doença e um atendimento multidisciplinar com profissionais de diversas áreas - pediatra, ortopedista, neurologista, geneticista, fisioterapeuta, psicólogo, nutricionista, assistente social, entre outros ${ }^{5,10}$.

Desse modo, a integralidade do cuidado associada à equidade são princípios do Sistema Único de Saúde (SUS), fundamentais para o seguimento do cuidado aos portadores de AMC. Uma vez que contempla todas as ações de promoção, tratamento e reabilitação e garante o acesso a todos os níveis de atenção, mediante a integração dos serviços, como descrito nos relatos que necessitaram de encaminhamento via MACC. Assim, é possível evidenciar o quão importante e necessário se faz o SUS para o diagnóstico e manejo dos portadores da desafiadora síndrome de AMC, visando o cuidado de forma coordenada e resolutiva ${ }^{16}$.

\section{REFERÊNCIAS BIBLIOGRÁFICAS}

1. Svartman C, Fucs PMMB, Kertzman PF, Kampe PA, Rosseti F. Artrogripose múltipla congênita - Revisão de 56 pacientes. Rev Bras Ortop [Internet]. 1995 [cited 2020 Mar 21];30(1/2):45-52. Available from: http://rbo.org. $\mathrm{br} /$ detalhes/719/pt-BR/artrogripose-multipla-congenita---revisao-de-56-pacientes-

2. Otto AW. Monstrum humanum extremitatibus incurvatus: monstrorum sexcentorum descripto anatomica in Vratislaviae Museum: Anatomico-Pathologieum Breslau 1841. Clin Orthop. 1985;194:(4):321-2.

3. Stern WG. Arthrogryposis multiplex congenita. JAMA [Internet].1923 [cited 2020 Mar 21];81(18):1507-10. Available from: https://jamanetwork.com/journals/ jama/article-abstract/236736.

4. Lowry RB, Sibbald B, Bedard T, Hall JG. Prevalence of multiple congenital contractures including arthrogryposis multiplex congenita in Alberta, Canada, and a strategy for classification and coding. Birth Defects Res A Clin Mol Teratol [Internet]. 2010 [cited 2020 Mar 21];88(12):1057-61. Available from: https://www.ncbi. $\mathrm{nlm}$.nih.gov/pubmed/21157886

5. Dahan-Oliel N, Cachecho S, Barnes D, Bedard T, Davison AM, Dieterich $K$, et al. International multidisciplinary collaboration toward an annotated definition of arthrogryposis multiplex congenita. Am J Med Genet C Semin Med Genet [Internet]. 2019 [cited 2020 Mar 21];181(3):288-99. Available from: https://www.ncbi. nlm.nih.gov/pmc/articles/PMC6771513/

6. Hall JG, Opitz JM, Reynolds JF. Analysis of Pena Shokeir phenotype. Am J Med Genet [Internet].1986 [cited 2020 Mar 21];25(1):99-117. Available from: https://www. ncbi.nlm.nih.gov/pubmed/3541610

7. Hall JG. Arthrogryposis (multiple congenital contractures): diagnostic approach to etiology, classification, genetics, and general principles. Eur ] Med Genet [Internet]. 2014 [cited 2020 Mar 21];57(8):464-72. Available from: https://europepmc.org/article/med/24704792

8. Kiefer J, Hall JG. Gene ontology analysis of Arthrogryposis (multiple congenital contractures). Am J Med Genet Part C [Internet]. 2019 [cited 2020 Mar 21];117. Available from: https://onlinelibrary.wiley.com/doi/ abs/10.1002/ajmg.c.31733

9. Kowalczyk B, Feluś J. Arthrogryposis: an update on clinical aspects, etiology, and treatment strategies. Arch Med Sci: AM S [Internet]. 2016 [cited 2020 Mar 
21];12(1):10-24. Available from: https://www.ncbi. nlm.nih.gov/pmc/articles/PMC4754365/

10. Krasniqi F, Salihu S, Krasniqi I, Pistulli E. Arthrogryposis Multiplex Congenita - Case Report. Am Res J Pediatr [Internet]. 2018 [cited 2020 Mar 21];2(1):1-5. Available from: https://www.arjonline.org/papers/arjpd/v2-i1/2.pdf

11. Hall JG, Reed SD, Driscoll EP, Opitz JM. Part I. Amyoplasia: A common, sporadic condition with congenital contractures. Am J Med Genet. 1983; 15(4):571-90

12. Quintans MDS, Barbosa PR, Lucena B. Artrogripose congênita múltipla. Rev Ped SOPER] [Internet]. 2017 [cited 2020 Mar 21];17(3):23-7. Available from: http://revistadepediatriasoperj.org.br/detalhe_artigo.asp?id=1025

13. Trindade JA, Freitas JS, Menzen L, Laux C, Barbosa LR, Cardoso MCAF. Speech-language pathology aspects in a pediatric case of head and neck arthrogryposis. CoDAS [Internet]. 2018 [cited 2020 Apr 05];30(2):e20170181. Available from: http:// www.scielo.br/scielo.php?script $=$ sci_arttext\&pi$\mathrm{d}=\mathrm{S} 2317-17822018000200401 \&$ Ing $=$ en.
14. Di Leo G, Pascolo P, Hamadeh K, Trombetta A, Ghirardo S, Schleef J, Barbi E, Codrich D. Gastrostomy Placement and Management in Children: A Single-Center Experience. Nutrients [Internet]. 2019 [cited 2020 Apr 05];11(7):1555. Available from: https://www.ncbi.nlm. nih.gov/pmc/articles/PMC6683077/

15. Alves PV, Zhao L, Patel PK, Bolognese AM. Arthrogryposis: Diagnosis and Therapeutic Planning for Patients Seeking Orthodontic Treatment or Orthognathic Surgery. J Craniofac Surg [Internet]. 2007 [cited 2020 Apr 05];18(4):838-43. Available from: https://journals. Iww.com/jcraniofacialsurgery/Abstract/2007/07000/ Arthrogryposis__Diagnosis_and_Therapeutic_Planning.23.aspx

16. Ministério da Saúde (Brasil), Secretaria de Atenção à Saúde, Departamento de Ações Programáticas Estratégicas. Política Nacional de Atenção Integral à Saúde da Criança: orientações para implementação. Brasília (DF); 2018 [cited 2020 Apr 25]. Available from: http://www. saude.pr.gov.br/arquivos/File/Politica_Nacional_de_ Atencao_Integral_a_Saude_da_Crianca_PNAISC.pdf

\section{Agradecimentos}

Agradecemos Dra. Marta Regina Clivati, neurologista pediátrica do Centro Clivati - Neurologia e Desenvolvimento e colaboradora da residência médica de pediatria do Centro Universitário Fundação Assis Gurgacz por conceder dados clínicos e colaborar na interpretação do caso 2, bem como ao Professor Rafael Lirio Bortoncello, médico radiologista do serviço Diagnose Clínica de Imagem, em Toledo e Professor de Radiologia e Diagnóstico por Imagem do curso de Medicina da Universidade Federal do Paraná - Campus Toledo, por conceder e colaborar com a interpretação das imagens radiográficas.

\section{Os autores declaram não haver conflito de interesse.}

\section{Financiamento Próprio}

Correspondência:

Dagna Karen Oliveira

dagnaoliveira2015@gmail.com

Editor:

Prof. Dr. Paulo Henrique Manso

Recebido em: 18/08/2020

Aprovado em: 08/12/2020 